\title{
Disentangling the intragroup HI in Compact Groups of galaxies by means of X3D visualization
}

\author{
Lourdes Verdes-Montenegro ${ }^{1}$, Frederic Vogt ${ }^{2}$, Claire Aubery ${ }^{3}$ \\ Laetitie Duret $^{4}$, Julián Garrido ${ }^{1}$, Susana Sánchez ${ }^{1}$, Min S. Yun ${ }^{4}$, \\ Sanchayeeta Borthakur ${ }^{5}$, Kelley Hess ${ }^{6}$, Michelle Cluver ${ }^{7}$, Ascensión \\ del Olmo ${ }^{1}$ and Jaime Perea ${ }^{1}$ \\ ${ }^{1}$ IAA-CSIC, Granada, Spain \\ email: lourdes@iaa.es, jgarrido@iaa.es,sse@iaa.es, chony@iaa.es, jaime@iaa.es \\ ${ }^{2}$ European Southern Observatory (ESO), Vitacura, Santiago, Chile \\ email: Frederic Vogt <frederic.vogt@alumni.anu.edu.au> \\ ${ }^{3}$ Aix-Marseille Universite (Stage L3MPCI), Marseille, France \\ email: claireaubery@aol.com, laeti.duret@orange.fr \\ ${ }^{4}$ UMass, Amherst, Massachusetts \\ email: myun@astro.umass.edu \\ ${ }^{5} \mathrm{JHU}$, Baltimore, Maryland \\ email: sanchayeeta@gmail.com \\ ${ }^{6}$ ASTRON, the Netherlands Institute for Radio Astronomy, Dwingeloo, the Netherlands \\ email: hess@astro.rug.nl \\ ${ }^{7}$ Department of Physics and Astronomy, University of the Western Cape, Robert Sobukwe \\ Road, Bellville, 7535, South Africa \\ email: michelle.cluver@gmail.com
}

\begin{abstract}
As an extreme kind of environment, Hickson Compact groups (HCGs) have shown to be very complex systems. HI-VLA observations revealed an intrincated network of HI tails and bridges, tracing pre-processing through extreme tidal interactions. We found HCGs to show a large HI deficiency supporting an evolutionary sequence where gas-rich groups transform via tidal interactions and ISM (interstellar medium) stripping into gas-poor systems. We detected as well a diffuse HI component in the groups, increasing with evolutionary phase, although with uncertain distribution. The complex net of detected HI as observed with the VLA seems hence so puzzling as the missing one. In this talk we revisit the existing VLA information on the HI distribution and kinematics of HCGs by means of X3D visualization. X3D constitutes a powerful tool to extract the most from HI data cubes and a mean of simplifying and easing the access to data visualization and publication via three-dimensional (3-D) diagrams.
\end{abstract}

Keywords. Galaxies, compact groups, galaxy evolution, visualization.

\section{Introduction}

HCGs (Hickson 1982) are isolated systems of 4-8 galaxies with densities similar to cluster cores, where pre-processing mechanisms are expected to be efficient. Star formation rate and $\mathrm{M}\left(\mathrm{H}_{2}\right)$ of $\mathrm{HCG}$ spirals (Verdes-Montenegro et al. 1998; Iglesias-Páramo \& Vilchez 1999) are similar to control samples, very likely due to their high HI deficiencies. Interferometric VLA observations of the HI in 16 Hickson Compact Groups (Verdes-Montenegro et al. 2001, VM01; Sulentic et al. 2001, Verdes-Montenegro et al. 2002, 2005ab, Williams et al. 2002, 2005) revealed an intrincated network of HI tails and bridges, tracing pre-processing through extreme tidal interactions. We proposed an 
evolutionary sequence in which groups seem to be evolving from a phase where the gas is located in the galaxy disks, to intermediate cases where the HI is mostly found in the intragroup medium, and finally into a stage where almost no HI is detected, neither in the galaxies nor in the intragroup medium, so inhibiting SF. What physical processes give rise to this extreme deficiency is still a mystery, despite of the numerous studies performed since this evolutionary model was proposed. Comparison of VLA imaging with high-quality single dish GBT observations (Borthakur et al. 2010, 2014) has provided evidence for the existence of a diffuse HI component missed by the VLA that increases with evolutionary stage, more consistent with tidal than with ram-pressure stripping, and spread in some cases over a velocity range of more than $1000 \mathrm{~km} / \mathrm{s}$. This suggests that slow evolution of tidal debris may lead to a final stage where the HI becomes faint and extended - hence escaping detection by current interferometers - being returned to the intragroup medium. However, the number of groups analysed in VM01 by means of resolved HI maps was not high enough to allow checking/confirming a potential correlation between the mass in HI tails and the degree of HI deficiency. This analysis was further complicated by the difficulty in separating the HI mass in tails from the associated to the galaxy disks.

Sensitivities in the referred VLA studies reach a maximum value of $\mathrm{N}(\mathrm{HI})=5 \times 10^{19}$ $\mathrm{cm}^{-2}$ (e.g. HCG 92 study with VLA C+CnB+D, 20" resolution, Sulentic et al. 2001, Williams et al. 2002). SKA1-MID will provide the required field of view $\left(30^{\prime}\right)$ with a highly improved sensitivity able to provide key information on the fate of gas in HCGs. While SKA1 starts observations, further progress can be made through a) studies at complementary wavelengths, b) preparatory work through SKA pathfinders, e.g. MeerKAT, and c) advanced visualization techniques allowing to improve the insight into the complex HI cubes of these strongly interacting systems. Here we present preliminary results on the relation between the ratio of $\mathrm{HI}$ mass in tails and HI deficiency based on: a) a larger VLA dataset, and b) the use of the X3D pathway (Vogt et al. 2015), both a powerful tool to extract the most from HI data cubes and as a mean of simplifying and easing the access to data visualization and publication via three- dimensional (3D) diagrams.

\section{The X3D pathway applied to the study of HCGs}

In Vogt et al. (2015) we have introduced the concept of the X3D pathway as a new approach to create, visualize, and share/publish multidimensional datasets using 3D diagrams. The X3D open-source (and ISO standard) 3D file format lies at the center of an advanced data visualization product tree that includes interactive HTML documents, 3D printing, high-end animations (and possibly interactive PDF documents). Many scripts and examples were included in that paper, designed as stepping stones, so that any interested user can customize them for specific datasets. In this work we have exploited the current possibilities offered by the X3D pathway in terms of visualization applied to HIVLA data of HCGs. One of the Python codes supplied with Vogt et al. (2015) has been adapted in order to ease visualization of several HCGs. Separation of different HI structures based on spatial and kinematical) information has been performed, so improving previous determinations based only on inspection of channel and moment maps.

\section{HI intragroup mass versus HI deficiency}

In order to quantify the amount of high surface brightness HI detected with the VLA located in intragroup features (e.g. tails, bridges, clumps), we have inspected HI-VLA data for HCG 2, 7, 15, 16, 23, 26, 30, 31, 33, 37, 40, 44, 48, 49, 58, 79, 88, 93, 95, 96, 97, 


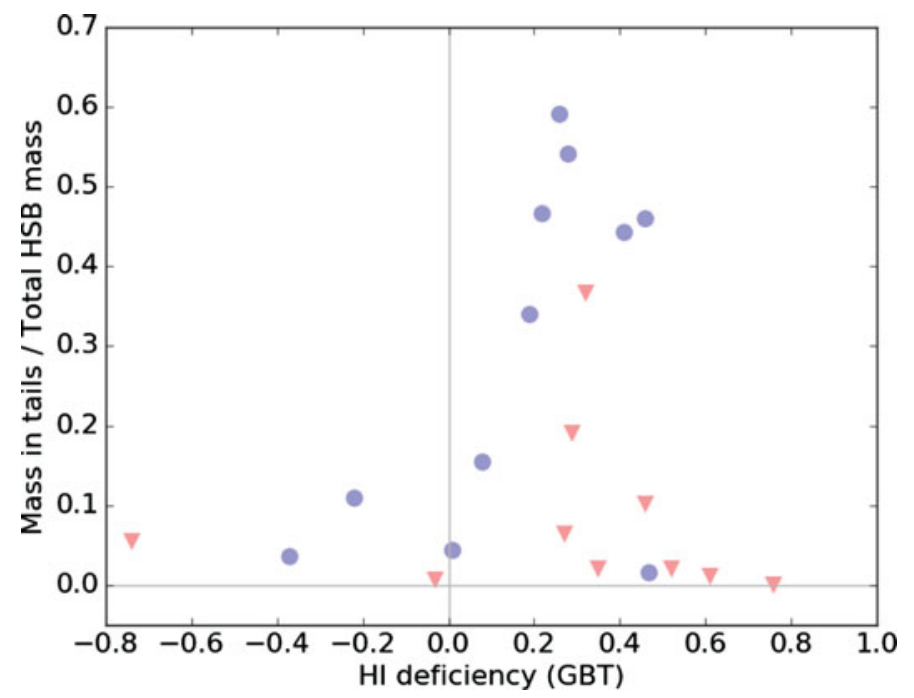

Figure 1. HI mass in the intragroup medium, divided by the total HI mass detected with the VLA, versus HI deficiency derived from GBT data. Blue dots: detections; pink triangles: upper limits

100 (own data and archive) with the X3D tool. The results are summarized in Fig. 1, where the ratio of mass in the intragroup medium, divided by the total HI mass detected with the VLA, is represented versus the total HI deficiency (it is, that derived from the HI masses obtained from single dish GBT, Borthakur et al. 2010). We remark that the measures represented in each axis are independent. The figure suggests that, if HCGs evolve from a normal HI content towards increasing HI deficiency (from left to right) they seem to do it via a phase of intensive tidal stripping. This might relate with results suggesting that MoHEG (Molecular Hydrogen Emiting Galaxy, Ogle 2010) are transition objects from the SF cloud to the quiescent cloud (Cluver et al. 2013) whose enhanced $\mathrm{H}_{2}$ emission might be energized by shocks caused by collisions within the cold intragroup medium. Improvement in the statistics by addition of further VLA data to the analysis is currently in preparation.

\section{References}

Borthakur, S., Yun, M., Verdes-Montenegro, L., Heckman, T. M., \& Zhu, G. 2014, AESAS 223, 328.01

Borthakur, S., Yun, M. S., \& Verdes-Montenegro, L. 2010, ApJ, 710, 385

Cluver, M. E., Appleton, P. N., Ogle, P., et al. 2013, ApJ, 765, 93

Hickson, P. 1982, ApJ, 255, 382

Iglesias-Páramo, J., \& Vilchez, J. 1999, ApJ, 518, 94

Ogle, P., Boulanger, F., Guillard, P., et al. 2010, ApJ, 724, 1193

Sulentic, J. W., Rosado, M., Dultzin-Hacyan, D., et al. 2001, AJ, 122, 2993

Verdes-Montenegro, L., Yun, M. S., Perea, J., del Olmo, A., \& Ho, P. T. P. 1998, ApJ, 497, 89

Verdes-Montenegro, L., Del Olmo, A., Iglesias-Páramo, J. I., et al. 2002, A\&̊A, 396, 815

Verdes-Montenegro, L., Del Olmo, A., Yun, M. S., \& Perea, J. 2005, A\&\&A, 430, 443

Verdes-Montenegro, L., Sulentic, J., Lisenfeld, U., et al. 2005, A\&\&A, 436, 443

Verdes-Montenegro, L., Yun, M. S., Williams, B. A., et al. 2001, A\& $A, 377,812$

Verdes-Montenegro, L., Del Olmo, A., Iglesias-Páramo, J. I., et al. 2002, A\&\&A, 396, 815

Vogt, F. P. A., Dopita, M. A., Borthakur, S., et al. 2015, MNRAS, 450, 2593

Williams, B. A., Yun, M. S., \& Verdes-Montenegro, L. 2002, AJ, 123, 2417 